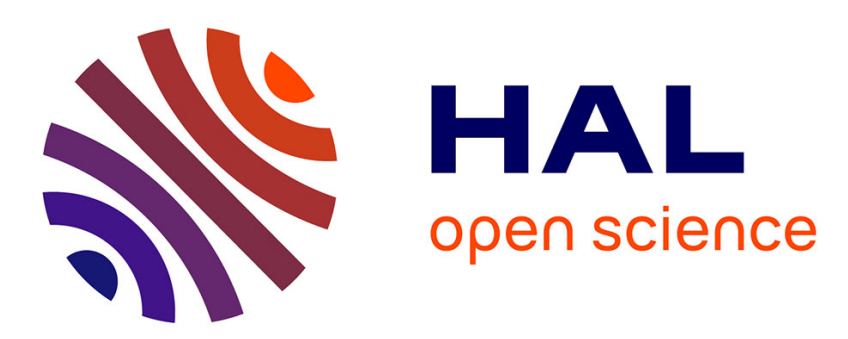

\title{
La gestion du résultat comptable autour d'un changement de dirigeant en France
}

\author{
Yves Mard, Sylvain Marsat
}

\section{To cite this version:}

Yves Mard, Sylvain Marsat. La gestion du résultat comptable autour d'un changement de dirigeant en France. Comptabilité - Contrôle - Audit, 2009, 10.3917/cca.153.0141 . hal-02156582

\section{HAL Id: hal-02156582 \\ https://hal.science/hal-02156582}

Submitted on 14 Jun 2019

HAL is a multi-disciplinary open access archive for the deposit and dissemination of scientific research documents, whether they are published or not. The documents may come from teaching and research institutions in France or abroad, or from public or private research centers.
L'archive ouverte pluridisciplinaire HAL, est destinée au dépôt et à la diffusion de documents scientifiques de niveau recherche, publiés ou non, émanant des établissements d'enseignement et de recherche français ou étrangers, des laboratoires publics ou privés. 


\title{
La gestion du résultat comptable autour d'un changement de dirigeant en France
}

\section{Earnings management surrounding CEO changes in France}

\author{
Yves MARD et Sylvain MARSAT* \\ Université d'Auvergne - Clermont Ferrand 1, \\ Faculté de Sciences Économiques et de Gestion, \\ IAE de Clermont Ferrand, \\ Centre de Recherches Clermontois en Gestion et Management (CRCGM), \\ 41 Boulevard François Mitterrand, 63002 Clermont-Ferrand Cedex, \\ E-Mail : yves.mard@u-clermont1.fr \\ sylvain.marsat@u-clermont1.fr
}

Version acceptée pour publication dans:

Mard, Y., Marsat, S., 2009. La gestion du résultat comptable autour d'un changement de dirigeant en France. Comptabilité Contrôle Audit numéro thématique, 141-170

\begin{abstract}
Résumé :
La gestion du résultat mise en œuvre par les dirigeants sortants et entrants est examinée à partir d'un échantillon de 52 changements de dirigeants au sein d'entreprises françaises cotées entre 2000 et 2005 . L'étude des accruals discrétionnaires montre d'abord que les dirigeants sortants ont tendance à gérer à la hausse les résultats durant les deux exercices qui précèdent leur départ. Ensuite, on observe une baisse très nette des accruals durant l'exercice de succession, conformément à l'hypothèse de "liquidation des pertes ». Durant les exercices qui suivent le changement, la remontée des accruals s'observe dès $\mathrm{T}+1$ en cas de changement planifié du dirigeant, mais seulement en $\mathrm{T}+2$ dans le cas d'un départ forcé. Enfin, une analyse multivariée montre, dans le contexte du changement de dirigeant, l'influence de certains mécanismes de gouvernance sur les variables comptables.
\end{abstract}

Mots-clés : Changements de dirigeant, gestion du résultat, départ planifié, départ forcé.

\begin{abstract}
:
We analyse earnings management carried out by the outgoing and the incoming top managers from a sample of 52 French firms who encountered a change of CEO during the 2000-2005 period. The study of discretionary accruals shows that they engage in upwards earnings management during the two years preceding their departure. Then, we observe a very clear decline of accruals during the year of succession, according to the "big bath" hypothesis. During the years following the change, upward earnings management is observed in case of routine changes as soon as $\mathrm{T}+1$, but only in $\mathrm{T}+2$ in the case of a non routine departure. Finally, a multivariate analysis pinpoints, in the case of CEO changes, the influence of some governance mechanisms on accruals.
\end{abstract}


Key words: CEO changes, earnings management, routine changes, non-routine changes.

* Les auteurs souhaitent remercier les participants au $29^{\text {ième }}$ Congrès de l'AFC (ESSEC, mai 2008), au

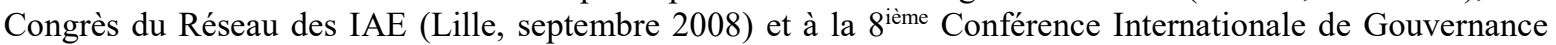
(Florence, juin 2009) pour leurs conseils et leurs suggestions, notamment Michel Albouy, Philippe Desbrières, Thomas Jeanjean, Julien Le Maux et Charles Piot. 


\section{Introduction}

Le changement de dirigeant constitue l'un des principaux mécanismes de gouvernance dont dispose le conseil d'administration d'une entreprise. Ces dernières années, les départs de dirigeants se sont intensifiés partout dans le monde. Par exemple, Karlsson et al. (2008) montrent qu'entre 1995 et 2007, le taux de changement parmi les plus grandes entreprises mondiales est passé de $9 \%$ à 14\%. En Europe, le phénomène est particulièrement marqué avec un taux de changement de $17,6 \%$ en 2007. Par ailleurs, la part de départs forcés a également fortement augmenté. En 2007, ils représentent près de la moitié des départs en Europe, ce que les auteurs attribuent aux réformes mises en œuvre en matière de gouvernance, telles que la loi de sécurité financière de 2003 en France.

Ces changements ont une implication sur la politique comptable des entreprises, notamment sur l'incitation pour les dirigeants à gérer le résultat comptable. L'objet de cette recherche est d'étudier cette gestion autour de l'exercice de succession. Plus précisément, nous analysons la gestion du résultat mise en œuvre par le dirigeant sortant avant son départ, puis la gestion du résultat opérée par le dirigeant entrant, pendant et après l'exercice de succession. Par ailleurs, nous étudions l'influence de la nature du départ (forcé ou planifié) sur les politiques comptables des dirigeants.

Tout d'abord, durant les années qui précèdent l'exercice de succession, le dirigeant sortant peut être incité à gérer à la hausse les résultats comptables (Pourciau, 1993). En cas de départ planifié, le dirigeant est tenté d'afficher un bilan et des résultats comptables flatteurs afin de maximiser son bonus de départ. En cas de départ forcé, faute de performance, le dirigeant peut tenter, dans une optique d'enracinement, de gérer le résultat pour sauver son poste et/ou sa réputation. 
Ensuite, pendant l'exercice de succession, le dirigeant entrant peut avoir intérêt à constater un maximum de charges sur l'exercice de succession. En effet, il fait alors porter la responsabilité des pertes sur son prédécesseur et préserve ainsi sa réputation (Murphy et Zimmerman, 1993). C'est la forme la plus testée de l'hypothèse de « liquidation des pertes » ${ }^{1}$.

Enfin, durant les années qui suivent l'exercice de succession, le dirigeant entrant va souhaiter afficher de bonnes performances afin d'asseoir sa position dans le poste. A cet effet, la liquidation des pertes opérée durant l'exercice de succession lui permet de repartir sur des bases saines. Par ailleurs, il peut être tenté d'amplifier la remontée mécanique des résultats en opérant des choix comptables discrétionnaires.

L'étude empirique porte sur les choix comptables effectués par les dirigeants sortants et entrants à partir d'un échantillon de 52 changements de dirigeant au sein d'entreprises françaises cotées entre 2000 et 2005 . Les résultats confirment que les dirigeants sortants ont tendance à gérer à la hausse les résultats durant les deux exercices qui précèdent leur départ. La gestion du résultat se concentre sur l'exercice T-2 en cas de départ forcé, alors qu'elle est maximale durant l'exercice T-1 juste antérieur au départ, lorsque le changement est planifié. Durant l'exercice $\mathrm{T}$ de succession, les accruals chutent fortement, conformément à l'hypothèse de «liquidation des pertes ». On constate par ailleurs que le phénomène est plus marqué en cas de départ forcé. Durant les exercices qui suivent l'exercice de succession, on observe une remontée des accruals discrétionnaires dès $\mathrm{T}+1$ en cas de départ planifié. En revanche, en cas de départ forcé, les accruals discrétionnaires restent largement négatifs en $\mathrm{T}+1$ avant de remonter nettement en $\mathrm{T}+2$. Enfin, une analyse multivariée confirme l'incidence de la nature du départ sur les variables comptables et montre l'influence de mécanismes de gouvernance tels que la composition du conseil de direction et de l'actionnariat. 
Cette recherche contribue à l'étude des politiques comptables à l'occasion des changements de dirigeants sur plusieurs points. Tout d'abord, les recherches portant sur les changements de dirigeants en France sont peu nombreuses ${ }^{2}$. L'étude du contexte du changement de dirigeant en France entre 2000 et 2005 nous a semblé intéressante, en raison de l'évolution des structures de gouvernance durant cette période, et de l'augmentation des départs, notamment forcés.

Ensuite, l'analyse distingue les départs planifiés et forcés, ce qui n'est le cas de la plupart des études qui portent sur les départs forcés (Pourciau, 1993; Guan et al., 2005), les départs planifiés (Reitenga et Tearney, 2003) ou ne distinguent pas les deux (Murphy et Zimmerman, 1993). D'autre part, l'analyse couvre la période $T-2$ à $T+2$ qui entoure le changement et s'intéresse aux dirigeants sortants et entrants. Notre étude est proche de celle de Wells (2002). Cependant, sur le plan méthodologique, l'utilisation d'un échantillon de contrôle et de tests multivariés complètent ses travaux.

Enfin, l'étude sur cinq années met en évidence une différence de configuration des variables comptables entre les deux types de départs, et montre l'influence des variables de gouvernance autour du changement. Ces résultats nous apparaissent originaux par rapport à l'ensemble des études déjà menées.

Cette étude comprend trois parties: la première présente la revue de littérature et les hypothèses de recherche, la deuxième décrit l'échantillon et la méthodologie adoptée, et enfin la troisième analyse les principaux résultats obtenus. 


\section{Problématique et hypothèses de la recherche}

L'étude des politiques comptables autour d'un changement de dirigeant conduit à s'interroger, d'une part, sur les motivations du dirigeant sortant, et d'autre part, sur celles du dirigeant entrant. Par ailleurs, la nature du départ (forcé ou non) et les caractéristiques de gouvernance de la firme sont susceptibles d'influencer les décisions comptables prises par les dirigeants dans ce contexte particulier.

\subsection{Les motivations du dirigeant sortant}

\subsubsection{Les justifications théoriques}

Pour le dirigeant sortant, les incitations à la gestion du résultat précédant un changement de dirigeant peuvent être liées à la crainte d'être démis en cas de faible performance, ou à une réduction de l'horizon temporel du dirigeant lorsque son départ est prévu.

Pour les dirigeants d'entreprise, le marché du travail peut présenter des opportunités au sein de la firme ou à l'extérieur (Fama, 1980). A l'inverse, ce marché exerce une certaine discipline sur les dirigeants par l'intermédiaire du conseil d'administration. En effet, en cas de faible performance, le dirigeant peut être démis de ses fonctions au profit d'un autre manager issu de l'entreprise (marché du travail interne) ou extérieur à la firme (marché du travail externe). La littérature met d'ailleurs en évidence une relation entre performance et changement de dirigeant. Il apparaît que la performance comptable influence significativement la probabilité de départ (Coughlan et Smith, 1985; Warner et al., 1988; Weisbach, 1988; Murphy et Zimmerman, 1993). Selon Hermalin et Weisbach (1998), les cours boursiers ne permettent seuls de juger de la performance du dirigeant car ils intègrent les attentes du marché concernant le devenir du dirigeant. Par conséquent, les comités de direction ont intérêt à utiliser des mesures comptables de la performance lorsqu'ils décident 
de l'avenir du dirigeant. Ce dernier peut donc être incité à gérer le résultat comptable afin de limiter le risque d'être démis de ses fonctions. Dans ce cas, la politique comptable appuie une stratégie d'enracinement du dirigeant, tout comme le font les investissements spécifiques dans l'approche développée par Shleifer et Vishny (1989).

Dans le contexte français, la théorie de l'enracinement a été mobilisée par plusieurs auteurs (Pichard-Stamford [1997], Alexandre et Paquerot [2000]). Ces derniers montrent l'inefficacité des structures de contrôle en France et l'expliquent par les différences entre le modèle français et le modèle anglo-saxon. L’enracinement spécifique des dirigeants français serait notamment la conséquence de l'absence de recours juridique des actionnaires à l'encontre des administrateurs, et des liens croisés qui existent au sein des conseils d'administration.

Concernant l'horizon temporel, l'augmentation du turnover des dirigeants des grandes entreprises à l'échelle mondiale les soumet à une pression grandissante. Elle les incite à une gestion à court terme des résultats, sous peine d'être contraints au départ. Par ailleurs, dans le cas d'un départ planifié du dirigeant, ce dernier voit son horizon temporel se réduire et peut être incité à prendre des décisions à court terme (Dechow et Sloan, 1991 ; Pourciau, 1993). En effet, dans ce contexte, les questions de réputation et de carrière étant marginales, le dirigeant ne sera a priori pas motivé pour s'investir fortement dans sa mission. Il est donc dans son intérêt de prendre certaines décisions comptables et financières afin de maximiser à court terme les résultats et sa prime de départ. Par exemple, le dirigeant sur le départ peut choisir de limiter certains investissements à long terme ou de minorer la prise en compte de certains risques, comme sous-estimer une provision. 


\subsubsection{Les résultats empiriques}

Plusieurs recherches empiriques obtiennent des conclusions conformes à l'hypothèse de gestion du résultat par les dirigeants avant leur départ. Dechow et Sloan (1991) ont mis en évidence que les managers sortants restreignent les dépenses de recherche et développement durant l'année qui précède leur remplacement. Plus récemment, Guan et al. (2005) étudient les départs forcés de dirigeants et mesurent des accruals discrétionnaires plus élevés l'année avant leur éviction. Des tests complémentaires semblent montrer que la performance et les caractéristiques des firmes ne suffisent pas à expliquer les résultats obtenus. Au contraire, ces analyses vont dans le sens d'une action délibérée des managers sur les chiffres comptables. L'étude de Reitenga et Tearney (2003) porte sur les changements planifiés de dirigeants. En étendant l'horizon d'analyse aux quatre années qui précèdent le départ, ils observent que les accruals discrétionnaires sont plus élevés lorsque le dirigeant garde un mandat d'administrateur après le changement. La présence d'investisseurs institutionnels semble aussi exacerber la gestion du résultat. A l'inverse, des facteurs de gouvernance tendent à l'atténuer, tels que l'indépendance du conseil d'administration et du comité d'audit, et l'actionnariat du dirigeant.

Les travaux de Murphy et Zimmermann (1993) conduisent à des résultats plus contrastés et, selon eux, les variables comptables sont davantage déterminées par la faible performance de l'entreprise que par la volonté des dirigeants. Les résultats obtenus par Pourciau (1993) sur un échantillon de départs non planifiés sont également mitigés. Contrairement à l'hypothèse formulée, les accruals discrétionnaires sont négatifs en T-1, année qui précède le changement de dirigeant. Néanmoins, dans la mesure où les accruals discrétionnaires sont positifs en T-2, l'auteure évoque le problème d'horizon temporel, selon lequel les managers ont peut-être entrepris la gestion du résultat dès T-2, les difficultés devenant impossibles à masquer en T-1. 
En Australie, Wells (2002) observe des accruals positifs durant l'année T-1, mais les valeurs obtenues ne sont pas significativement différentes de zéro. Enfin, en Grande-Bretagne, Conyon et Florou (2006) n'observent pas de restriction des dépenses de recherche et développement antérieures à un changement de dirigeant. Par conséquent, les résultats des études empiriques ne confirment pas unanimement l'hypothèse selon laquelle le dirigeant sortant tend à augmenter le résultat comptable.

Cependant, compte tenu du cadre théorique énoncé, et conformément à l'hypothèse formulée dans les études antérieures (Murphy et Zimmerman, 1993 ; Dechow et Sloan, 1991 ; Reitenga et Tearney ; 2003 ; Guan et al., 2005), nous supposons tout d'abord, que les dirigeants ont tendance à gérer à la hausse le résultat comptable avant leur remplacement :

Hypothèse 1: Durant les exercices précédant son départ, le dirigeant sortant a tendance à gérer le résultat comptable à la hausse.

\subsection{Les motivations du dirigeant entrant}

\subsubsection{Les justifications théoriques}

Pour le dirigeant entrant, la volonté de ne pas décevoir les différentes parties prenantes à la firme se traduit par une obligation de performance, et donc de résultat. En finance d'entreprise, plusieurs théories ont été avancées pour expliquer l'impact d'un nouveau dirigeant sur la performance de l'entreprise (Caby et Hirigoyen, 2005):

- la théorie du «bon sens », selon laquelle le changement de dirigeant permet d'améliorer la performance de la firme (Guest, 1962), 
- la théorie du «bouc émissaire », selon laquelle le changement n'a pas d'impact sur la performance, mais vise à rassurer les parties prenantes à la firme sur sa bonne gouvernance (Gamson et Scotch, 1964),

- la théorie du « cercle vicieux », selon laquelle le changement de dirigeant tend à déstabiliser l'entreprise et à diminuer encore la performance ${ }^{3}$.

Dans ce contexte d'incertitude sur l'évolution de la performance de la firme suite à l'arrivée du nouveau dirigeant, ce dernier va devoir afficher de bons résultats durant les premières années de son mandat. Il pourra ainsi conserver son poste et préserver sa réputation. Selon Fama (1980), le capital humain constitue la meilleure arme dont dispose un manager pour bénéficier d'opportunités de carrières (internes ou externes) sur le marché du travail. De fait, la carrière d'un dirigeant est largement influencée par sa réputation, c'est-à-dire la façon dont est perçue sa capacité à bien gérer une entreprise et à obtenir des résultats. En particulier, lorsqu'un dirigeant arrive à la tête d'une entreprise, ses premières décisions et les premiers résultats obtenus font l'objet d'une attention particulière de la part des différentes parties prenantes à la firme. Parmi les indicateurs de performance, le résultat comptable constitue une variable qui peut influencer la réputation d'un dirigeant. Ce dernier est donc incité à gérer les chiffres comptables durant les premiers exercices de son mandat dans le but de maximiser sa réputation et la probabilité de rester en poste, même si une politique comptable trop agressive peut être coûteuse en termes de réputation (Desai et al., 2006).

Afin d'optimiser sa stratégie de gestion des résultats comptables, le dirigeant entrant peut être incité à enregistrer un maximum de pertes sur l'exercice de succession (De Angelo, 1988). En effet, cet exercice de transition est en général un exercice partiel et le dirigeant entrant pourra aisément attribuer au sortant la responsabilité des mauvais résultats enregistrés durant cet exercice. Par ailleurs, du fait de la réversibilité des chiffres comptables, le dirigeant entrant 
bénéficie ensuite de la remontée mécanique des résultats durant les exercices qui suivent l'exercice de succession. Ce regain de performance permet au nouveau dirigeant de renforcer sa réputation (Murphy et Zimmermann, 1993).

\subsubsection{Les résultats empiriques}

Moore (1973) a été un des premiers à tester l'hypothèse de liquidation des pertes. Sa recherche met en évidence une proportion plus forte de décisions comptables discrétionnaires ayant un impact négatif sur le résultat parmi les entreprises qui ont changé de dirigeants. De Angelo (1988) a analysé 86 tentatives de prise de contrôle entre 1970 et 1983. Elle observe qu'en cas d'élection des dissidents, ces derniers «chargent» les résultats en accablant les dirigeants vaincus. De même, Lasalle (1990) constate un lien entre les changements de dirigeants et les accruals ayant un effet négatif sur le résultat. Une étude de Pourciau (1993) semble également confirmer cette hypothèse. A partir d'un échantillon de 73 changements de dirigeants, elle constate que les nouveaux dirigeants ont tendance à prendre des décisions comptables diminuant les résultats de l'exercice durant lequel s'est fait le changement. De même, Francis, Hanna, et Vincent (1996) observent, après contrôle de la performance, des dotations aux provisions (pour dépréciation et pour charges) plus fréquentes et plus élevées parmi les entreprises ayant récemment changé de dirigeant. Enfin, Wells (2002) vérifie l'hypothèse de liquidation des pertes dans le cas de changements forcés de dirigeants.

Plusieurs recherches aboutissent à des résultats plus contrastés. Ainsi, Lasalle, Jones et Jain (1993) étudient les décisions comptables (en dehors des diminutions de valeur des actifs) de 92 entreprises. Ils constatent que les entreprises ayant changé de dirigeant effectuent davantage de modifications comptables que les autres. Ce résultat peut s'expliquer par le fait que les dirigeants entrants ont une vision de l'entreprise différente de celle des dirigeants sortants. Cependant, les modifications n'ont pas une probabilité plus élevée d'entraîner une 
baisse des résultats qu'une hausse des résultats, et ce quelque soit la performance de l'entreprise. Murphy et Zimmerman (1993) obtiennent aussi des résultats mitigés en analysant le comportement d'un certain nombre de variables comptables à l'occasion de changements de dirigeants. Ils observent que les modifications de ces variables, qui se traduisent par un impact négatif sur les résultats, s'expliquent essentiellement par la faiblesse des performances de l'entreprise. Cependant, la majorité des études empiriques tendent à confirmer l'hypothèse de liquidation des pertes dans différents contextes. Cela nous conduit à formuler une seconde hypothèse :

Hypothèse 2 : Durant l'exercice de succession, le dirigeant entrant a tendance à gérer le résultat comptable à la baisse.

Enfin, une troisième hypothèse concerne les exercices suivant l'exercice de succession, durant lesquels on s'attend à une gestion à la hausse du résultat. L'étude de Wells (2002) ne semble pas aller dans ce sens. En revanche, les recherches conduites par Pourciau (1993) et Godfrey et al. (2003) accréditent cette hypothèse ${ }^{4}$. Aussi, en accord avec les hypothèses des études antérieures, nous supposons que les dirigeants vont alors gérer à la hausse les résultats comptables :

Hypothèse 3 : Après l'exercice de succession, le dirigeant entrant a tendance à gérer le résultat comptable à la hausse.

\subsection{L'influence de la nature du changement de dirigeant}

La nature du départ (planifié ou forcé) peut influencer significativement les motivations des dirigeants. Dans le cas d'un départ forcé, celui-ci est souvent lié à la faiblesse des performances de l'entreprise. On peut donc s'attendre à une incitation à la gestion haussière 
du résultat plus forte que dans le cas d'un départ planifié (Murphy et Zimmerman, 1993 ; Wells, 2002).

De même, pour le dirigeant entrant, on peut penser que les attentes des parties prenantes à la firme sont plus fortes dans le cas d'un départ forcé, qu'à l'occasion d'un départ planifié. L'incitation à liquider les pertes durant l'exercice de succession, et à gérer ensuite le résultat à la hausse, n'en est que plus grande. Par exemple, Murphy et Zimmerman (1993) constatent que les dirigeants entrants semblent utiliser leur latitude principalement parmi les entreprises dont le changement a été motivé par la médiocrité des performances. De même, Pourciau (1993) et Wells (2002) vérifient l'hypothèse de liquidation des pertes dans le cas des changements forcés. Ces derniers résultats conduisent à penser que le phénomène de « liquidation des pertes » pourrait être amplifié en cas de départ forcé. Nous formulons donc l'hypothèse générale suivante :

Hypothèse $4:$ La gestion du résultat mise en œuvre par les dirigeants entrants et sortants est plus importante dans le cas d'un départ forcé que dans le cas d'un départ planifié.

Cette hypothèse peut se décliner en trois sous-hypothèses correspondant aux trois périodes étudiées, c'est-à-dire avant, pendant et après l'exercice de succession :

Hypothèse 4a : Durant les exercices précédant son départ, la gestion du résultat à la hausse par le dirigeant sortant est plus importante dans le cas d'un départ forcé que dans le cas d'un départ planifié.

Hypothèse 4b : Durant l'exercice de succession, la gestion du résultat à la baisse par le dirigeant entrant est plus importante dans le cas d'un départ forcé que dans le cas d'un départ planifié. 
Hypothèse 4c : Après l'exercice de succession, la gestion du résultat à la hausse par le dirigeant entrant est plus importante dans le cas d'un départ forcé que dans le cas d'un départ planifié.

\subsection{L'influence des caractéristiques de gouvernance}

Afin de compléter l'analyse et de mieux comprendre les déterminants de la gestion des variables comptables, nous nous intéressons maintenant aux facteurs de gouvernance susceptibles d'influencer les choix opérés par le dirigeant. Parmi les caractéristiques de gouvernance, nous distinguons celles qui concernent les organes de direction, la composition de l'actionnariat et les caractéristiques des dirigeants.

\section{Organes de direction}

Tout d'abord, la forme du conseil de direction va déterminer la séparation entre les fonctions de direction et les fonctions de contrôle. La séparation est effective dans le cas d'un conseil de direction organisé sous la forme d'un conseil de surveillance et d'un directoire, et dans le cas d'un conseil d'administration où les fonctions de président et de directeur général sont dissociées.

D'autre part, la structure même du conseil de direction peut influencer, de par le nombre d'administrateurs indépendants entrant dans sa composition, les décisions prises par le dirigeant, en particulier en matière comptable (Peasnell, Pope et Young, 2005).

\section{Composition de l'actionnariat}

La composition de l'actionnariat peut également agir comme un mécanisme de gouvernance. La concentration du capital constitue une garantie de bonne gouvernance, même si certains auteurs soulignent une relation non monotone entre la répartition de l'actionnariat et la qualité 
de l'information financière (Labelle et Schatt, 2005 ; Bozec, 2008). En particulier, lorsque les dirigeants sont actionnaires, l'intérêt du principal et de l'agent convergent, ce qui devrait discipliner le dirigeant dans sa présentation des comptes. Cependant, ce dernier peut être incité, selon la théorie de l'enracinement, à agir dans son intérêt au détriment des actionnaires minoritaires (Fama et Jensen, 1983).

\section{Caractéristiques des dirigeants}

Selon Reitenga et Tearney (2003), l'incitation à la gestion du résultat est supérieure lorsque le dirigeant souhaite conserver un poste d'administrateur au sein de l'entreprise. Par ailleurs, les dirigeants détenteurs de stock-options peuvent aussi être incités à gérer à la hausse les résultats afin d'influencer le cours des actions et lever leurs options. Selon les circonstances, et afin de maximiser sa plus-value, le dirigeant est incité à diminuer le résultat avant l'attribution de stock-options, ou au contraire à augmenter le résultat avant de les exercer (Aboody et Kasznik, 2000). L'incitation des dirigeants à agir sur les résultats lorsqu'ils détiennent des stock-options a été mise en évidence par Bergstresser et Philippon (2006) et Burns et Kedia (2006).

Nous formulons donc l'hypothèse suivante :

Hypothèse 5 : Les facteurs de gouvernance influencent les variables comptables autour du changement de dirigeant. 


\section{Echantillons et méthodologie}

Nous présentons maintenant les échantillons utilisés et la mesure de la gestion du résultat retenue. Ensuite, nous exposons l'opérationnalisation des variables concernant la nature du changement de dirigeant, ainsi que celles de gouvernance et de contrôle.

\subsection{Les échantillons sélectionnés}

Les comptes consolidés des entreprises françaises non financières ${ }^{5}$ de l'indice SBF 250 , entre 2000 et 2005, ont été étudiés. Les données comptables utilisées proviennent de la base de données Reuters et des rapports annuels des sociétés. L'analyse nécessite deux échantillons différents : celui des sociétés ayant connu un changement de dirigeant et un échantillon de contrôle.

\section{- L'échantillon des sociétés ayant changé de dirigeant}

Au total, 52 changements de dirigeant entre 2000 à 2005 ont été analysés ${ }^{6}$. Le changement du responsable du document de référence, qui est censé se porter garant des choix comptables de sa société, a permis de déterminer précisément la date du renouvellement. Par rapport à l'exercice T-1, l'exercice $\mathrm{T}$ de la succession se caractérise donc par un nouveau responsable du document de référence. La répartition des années de changements de dirigeants figure dans le tableau 1.

Tableau 1

Années de changement de dirigeant des sociétés étudiées $(\mathrm{N}=52)$

\begin{tabular}{|cccccc|}
\hline Année & 2001 & 2002 & 2003 & 2004 & 2005 \\
\hline Nombre de changements de dirigeant & 2 & 9 & 11 & 21 & 9 \\
\hline
\end{tabular}


De plus, l'analyse a été approfondie en répartissant les entreprises en 6 secteurs d'activité d'après la classification retenue par la base de données Reuters ${ }^{7}$.

\section{- L'échantillon de contrôle}

L'échantillon de contrôle comprend 180 sociétés, provenant elles aussi de l'indice SBF 250. Naturellement, les sociétés de l'échantillon de changement de dirigeants ont été retirées de l'échantillon de contrôle. Cet échantillon est représentatif de la population des sociétés françaises durant cette période si l'on écarte les sociétés financières et celles ayant fait faillite. Les données extrêmes ont été exclues de la régression afin d'éviter une estimation biaisée des coefficients. Le nombre total d'observations s'élève ainsi à 508 données, soit 180 entreprises sur 3 ans, auquel on ôte 22 données manquantes et 10 extrêmes ${ }^{8}$.

En termes de performance, les sociétés de l'échantillon de contrôle affichent en moyenne des ROA et ROE sur la période 2001-2005 supérieures à ceux de l'échantillon d'étude, comme le montre le tableau 2 ci-dessous.

Tableau 2

Moyenne des ROA et ROE médians des échantillons

\begin{tabular}{lc}
\hline & Moyenne 2001-2005 \\
\hline ROA médian de l'échantillon de contrôle & $3,60 \%$ \\
ROA médian de l'échantillon d'étude & $2,29 \%$ \\
ROE médian de l'échantillon de contrôle & $13,36 \%$ \\
ROE médian de l'échantillon d'étude & $10,39 \%$ \\
\hline
\end{tabular}

Cet écart peut s'expliquer à la fois par les différences de composition des deux échantillons du point de vue de la taille et du secteur d'activité, ainsi que par le contexte de faible performance qui accompagne souvent le changement de dirigeant. 


\subsection{La mesure de la gestion du résultat}

Le montant des accruals, différence entre le bénéfice net et les flux de trésorerie d'exploitation (cash-flow) apparaît comme la variable la plus pertinente pour l'étude de la gestion du résultat à partir des décisions comptables. Les accruals regroupent les éléments calculés et décalés du résultat, comme la production immobilisée, les amortissements et provisions, ou encore la variation du besoin en fonds de roulement. Ces postes constituent des instruments privilégiés de gestion du résultat, car les dirigeants disposent d'une certaine latitude dans leur évaluation. Les mesures de la gestion du résultat à partir des accruals consistent à extraire des accruals totaux la partie laissée à la discrétion des dirigeants (accruals discrétionnaires).

\subsubsection{Calcul des accruals totaux}

Les accruals totaux ont été calculés de la façon suivante :

Accruals totaux $=$ Résultat net - Flux de trésorerie d'exploitation.

Le montant total des accruals $\mathrm{AT}_{\mathrm{i}, \mathrm{t}}$ de la firme i pour l'année t a été normalisé à travers le rapport $\mathrm{ATN}_{\mathrm{i}, \mathrm{t}}=\mathrm{AT}_{\mathrm{i}, \mathrm{t}} / \mathrm{A}_{\mathrm{i}, \mathrm{t}-1}$, où $\mathrm{A}_{\mathrm{i}, \mathrm{t}-1}$ est le montant total de l'actif de la firme $\mathrm{i}$ pour l'année t-1.

\subsubsection{Le modèle général d'extraction des accruals discrétionnaires}

Le modèle adopté s'inscrit dans le prolongement de ceux de Jones (1991) et Dechow (1994). Les amortissements ont néanmoins été éliminés des accruals totaux car, comme le souligne Beneish (1998), leur manipulation apparaît difficile. En effet, diverses recherches (e.g. Teoh et al., 1998) ne retiennent que la partie court terme des accruals (variation du besoin en fonds de roulement) pour le calcul des accruals discrétionnaires. Seuls les amortissements ont été enlevés, afin de conserver les provisions parmi les accruals, dans la mesure leur évaluation 
bénéficie d'une certaine latitude. Les accruals retenus regroupent donc essentiellement la variation du besoin en fonds de roulement et celle des provisions d'exploitation. Les statistiques de ces accruals pour l'échantillon de contrôle sont présentées dans le tableau 3. L'étude des accruals totaux (hors dotations aux amortissements) normalisés des entreprises de notre échantillon de contrôle indique que ces accruals sont en moyenne faiblement négatifs, sauf en 2003, année où la conjoncture économique fut défavorable.

Tableau 3

Moyenne et médiane des accruals totaux (hors dotations aux amortissements) pour l'échantillon de contrôle $(\mathrm{N}=508)$

\begin{tabular}{|llll}
\hline & $\mathbf{2 0 0 2}$ & $\mathbf{2 0 0 3}$ & $\mathbf{2 0 0 4}$ \\
\hline Moyenne & $-0,06 \%$ & $-0,43 \%$ & $-0,07 \%$ \\
Médiane & $-0,05 \%$ & $-0,73 \%$ & $-0,21 \%$ \\
\hline
\end{tabular}

Le modèle de base d'estimation des accruals d'une entreprise i pour l'année t est le suivant :

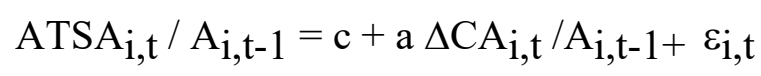

où :

ATSA $_{i, t}$ est le montant total des accruals totaux (hors dotations aux amortissements) de la firme i pour l'année t;

$A_{i, t-1}$ est le montant total de l'actif net de la firme i en fin d'année t-1;

$\Delta \mathrm{CA}_{\mathrm{i}, \mathrm{t}}$ est égal à la différence entre le chiffre d'affaires de l'année $\mathrm{t}$ et le chiffre d'affaires de l'année t-1 pour la firme i;

a et $\mathrm{c}$ sont des paramètres à déterminer;

$\varepsilon_{\mathrm{i}, \mathrm{t}}$ est le terme d'erreur de la régression.

$\varepsilon_{\mathrm{i}, \mathrm{t}}$ correspond donc à une estimation des accruals discrétionnaires normalisés par l'actif de l'entreprise i pour l'année t. Il mesure ainsi les accruals non expliqués par l'activité 
« normale » de l'entreprise, comme ceux nécessaires à la variation du besoin en fonds de roulement ou aux dotations aux provisions.

\subsubsection{Calcul des accruals discrétionnaires}

Sur la période de référence 2002-2004, les coefficients a et c ont été estimés à partir de l'échantillon de contrôle. Afin d'éviter tout problème d'hétéroscédasticité dans l'estimation des accruals discrétionnaires, les données ont été normalisées par la valeur de l'actif.

Deux approches différentes ont été utilisées pour mesurer les coefficients a et c qui permettent d'évaluer les accruals discrétionnaires: soit (1) regrouper dans la régression toutes les observations de l'échantillon de contrôle sur la période 2002-2004 (508 observations) ou soit (2) effectuer des régressions par secteur d'activité (6 secteurs retenus), ce qui revient à calculer des coefficients as et cs pour chaque secteur d'activité $(S=1, \ldots 6)$, dans la lignée du modèle développé par DeFond et Jiambalvo (1994).

\subsection{La nature du changement de dirigeant}

La nature du départ, forcé ou non, a été déterminée en examinant méthodiquement les rapports annuels et des articles publiés dans la presse économique. La révocation est peu souvent explicitée. Farrell et Whidbee (2003) et Engel et al. (2003) proposent une classification des changements de dirigeant qui distingue les départs forcés et non forcés. Aussi, nous avons considéré comme forcés les départs où il apparaît que les dirigeants ont été poussés vers la sortie pour des raisons de changement de contrôle, de différend politique, de faible performance ou encore de scandale. La faiblesse des résultats des entreprises est souvent prédominante. C'est le cas, par exemple, pour les dirigeants de Bacou-Dalloz, Canal Plus, Club Med, Eurotunnel, Rhodia, ou Vivendi. Certains départs font suite à des affaires financières ou à des crises (Otor, Buffalo Grill) ayant entraîné des difficultés pour l'entreprise. 
D'autres, comme Carrefour, sont la conséquence de tensions entre la direction et les actionnaires.

Les changements pour lesquels le dirigeant est parti de son propre gré constituent, en revanche, les départs non forcés. C'est le cas des départs en retraite, des promotions internes ou externes, ou des départs pour des raisons de maladie. Le cas le plus fréquent est celui des départs en retraite ou en préretraite (par exemple Air Liquide, Cap Gemini, Gaumont, Renault...). D'autre part, nous avons considéré que la promotion d'un dirigeant vers d'autres fonctions constituait un départ non forcé. Celle-ci peut s'effectuer au sein du groupe (Eurodisney, GDF) ou à l'extérieur (le dirigeant de France Télécom s'est vu proposer une responsabilité politique, par exemple).

En conclusion, compte tenu de la partition retenue, il apparaît parmi les départs forcés une majorité de départs liés à la faible performance de l'entreprise, contexte propice à la gestion du résultat comptable. Par ailleurs, parmi les départs non forcés, la plupart correspondent à des départs en retraite planifiés, susceptibles également de conduire à la gestion à court terme du résultat, du fait de la réduction de l'horizon temporel des dirigeants. Au total, sur les 52 changements de dirigeants, 26 correspondent à des départs forcés et l'autre moitié à des départs planifiés.

\subsection{Les variables de gouvernance et de contrôle}

Afin d'opérationnaliser les variables de gouvernance, nous avons utilisé des données issues des rapports annuels. Les variables de contrôle sont relatives aux caractéristiques de la firme (taille, endettement, performance, choix comptables antérieurs). En effet, la théorie politicocontractuelle de la comptabilité, développée par Watts et Zimmerman (1986), suggère que la 
taille (coûts politiques) et l'endettement (coûts contractuels) influencent les politiques comptables des sociétés. Par ailleurs, Dechow et al. (1995) constatent que les modèles d'estimation des accruals discrétionnaires peuvent être mal spécifiés pour les firmes dont les performances sont extrêmes. La variable flux de trésorerie d'exploitation est donc introduite comme variable de contrôle, à l'instar de Maijoor et Vanstraelen (2006). Enfin, les accruals discrétionnaires de l'année précédente sont intégrés à la régression afin de contrôler la réversibilité des variables comptables (Hunt, Moyer et Shevlin, 1996). L'opérationnalisation et les statistiques descriptives des variables, sur la période complète $\mathrm{T}-2$ à $\mathrm{T}+2$, sont présentées avec le tableau $4^{9}$.

Tableau 4

Statistiques descriptives des variables de gouvernance et de contrôle $(\mathrm{N}=52)$

\begin{tabular}{|cccccccc|}
\hline Variable \ Année & & [T-2, T+2] & T-2 & T-1 & T & T+1 & T+2 \\
\hline Accruals discrétionnaires & AD & $0,01 \%$ & $0,69 \%$ & $0,56 \%$ & $-1,28 \%$ & $-0,40 \%$ & $0,50 \%$ \\
\hline Nature du départ & DEP & $50,0 \%$ & $50,0 \%$ & $50,0 \%$ & $50,0 \%$ & $50,0 \%$ & $50,0 \%$ \\
\hline Séparation direction et contrôle & SEP & $41,2 \%$ & $59,6 \%$ & $59,6 \%$ & $30,8 \%$ & $26,9 \%$ & $28,8 \%$ \\
Contrôle par les administrateurs & ADM & $44,0 \%$ & $43,9 \%$ & $43,3 \%$ & $41,5 \%$ & $43,9 \%$ & $47,2 \%$ \\
\hline Contrôle par les actionnaires & ACT & $49,5 \%$ & $52,1 \%$ & $51,6 \%$ & $49,2 \%$ & $48,5 \%$ & $46,1 \%$ \\
Contrôle par les dirigeants & DIR & $4,6 \%$ & $5,9 \%$ & $5,9 \%$ & $3,8 \%$ & $3,8 \%$ & $3,7 \%$ \\
\hline Situation ultérieure & SIT & $40,38 \%$ & $40,38 \%$ & $40,38 \%$ & $40,38 \%$ & $40,38 \%$ & $40,38 \%$ \\
Détention de stock-options & OPT & $72,3 \%$ & $61,5 \%$ & $61,5 \%$ & $76,9 \%$ & $78,8 \%$ & $82,7 \%$ \\
\hline Taille & TAIL & 21,7 & 21,7 & 21,7 & 21,7 & 21,8 & 21,8 \\
Endettement & END & $28,8 \%$ & $29,5 \%$ & $29,7 \%$ & $29,2 \%$ & $29,3 \%$ & $26,4 \%$ \\
Performance & FTE & $6,58 \%$ & $5,96 \%$ & $5,54 \%$ & $7,30 \%$ & $6,73 \%$ & $7,37 \%$ \\
\hline
\end{tabular}

AD est le montant des accruals discrétionnaires calculé à partir du modèle global, DEP est égal à 1 en cas de départ forcé, et à 0 sinon, SEP est égal à 0 si les organes de contrôle et de gestion sont séparés, 1 sinon, ADM est le pourcentage d'administrateurs indépendants dans le conseil de direction, ACT est le pourcentage de droits de vote détenu par les trois principaux actionnaires, DIR est le pourcentage de droits de vote détenu par le dirigeant, SIT est égal à $1 \mathrm{si}$ le dirigeant conserve un poste d'administrateur après le changement, 0 sinon, OPT est égal à $1 \mathrm{si}$ le dirigeant bénéficie d'options de souscription d'actions, 0 sinon, TAIL est le logarithme népérien du total de l'actif en début d'exercice, END est le rapport dettes financières long terme en fin d'exercice divisé par l'actif total en début d'exercice, FTE est le montant des flux de trésorerie d'exploitation de l'exercice divisé par l'actif total de début d'exercice.

Comme nous l'avions noté, la moitié des départs est forcée. Il est intéressant de remarquer un renforcement des structures de direction durant la période, avec une augmentation de la 
séparation entre direction et contrôle (de 40 à plus de 70\%) et de la part d'administrateurs indépendants (de 44 à 47\%). Le changement de dirigeant semble avoir été propice à la mise en œuvre de meilleures pratiques en matière de gouvernance dans la lignée des recommandations émises par la loi sur les Nouvelles Régulations Économiques (2001). Concernant la composition de l'actionnariat, la part détenue par les principaux actionnaires et le dirigeant décline durant la période, respectivement de 52 à $46 \%$ et de 5,9 à $3,7 \%$. Enfin, $40 \%$ des dirigeants conservent un poste d'administrateur et ils sont de plus en plus nombreux à détenir des stock-options (de 62 à $83 \%)$.

\section{Les résultats de l'étude empirique}

Nous présentons d'abord l'évolution des accruals totaux et discrétionnaires durant la période $\mathrm{T}-2$ à $\mathrm{T}+2$ qui entoure le changement de dirigeant. Sur cette période, nous analysons si les variables comptables diffèrent significativement d'un exercice sur l'autre. Ensuite, les accruals sont comparés à ceux mesurés au sein de l'échantillon de contrôle. Afin d'approfondir ces analyses, nous distinguons les départs selon leur nature (forcé ou non) et procédons à une régression introduisant des variables de gouvernance et de contrôle.

\subsection{Analyse longitudinale des accruals autour du changement de dirigeant}

La figure 1 permet de visualiser l'évolution de la moyenne des accruals discrétionnaires des sociétés étudiées pour les années $\mathrm{T}-2$ à $\mathrm{T}+2$ entourant le changement du dirigeant, dont la dernière année de responsabilité est l'année T-1. Sur la figure 1 apparaissent aussi les valeurs mesurées à partir des deux sous-échantillons relatifs aux départs forcés et aux départs planifiés, ainsi que la moyenne des accruals discrétionnaires mesurés sur l'échantillon de contrôle. 
Figure 1

Evolution des accruals discrétionnaires autour du changement de dirigeant

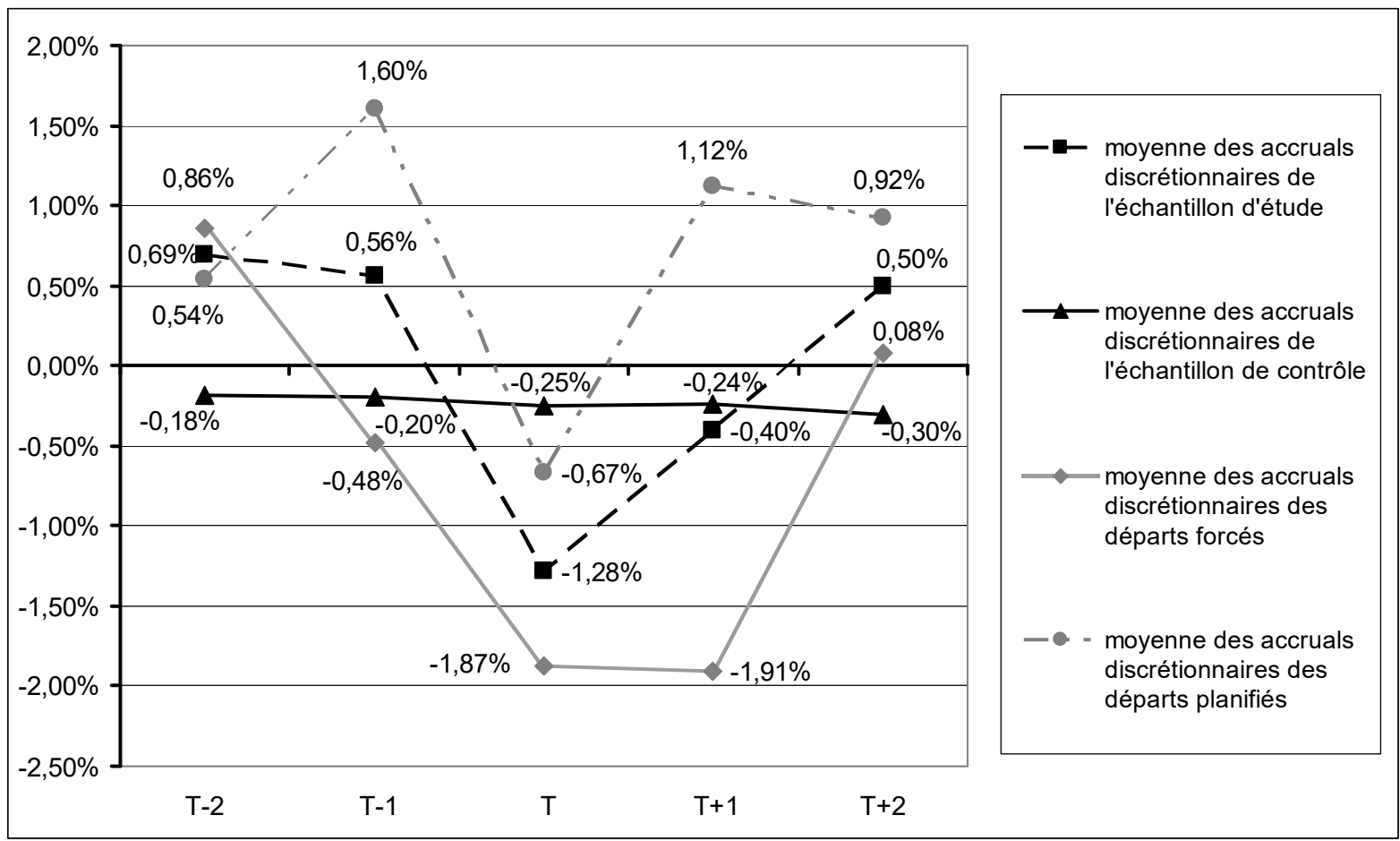

De façon plus détaillée, le tableau 5 présente les valeurs des accruals totaux et discrétionnaires durant cette période et les tests paramétriques et non paramétriques de l'analyse longitudinale. Les résultats indiquent une baisse des accruals l'année $\mathrm{T}$ du changement de dirigeant par rapport aux années T-2 et T-1. Les tests paramétriques (au seuil de 5\%) et non paramétriques (au seuil de 10\%) montrent une différence significative entre les valeurs observées avant le changement (T-2 et T-1) et les valeurs observées l'année $\mathrm{T}$ du changement. En analysant les résultats selon la nature du départ, on constate que dans le cas des départs forcés, la chute des accruals a lieu dès l'année T-1, alors qu'elle se fait en $\mathrm{T}$ dans le cas des départs planifiés, comme l'indique la figure 1. Ces résultats sont cohérents avec ceux obtenus par Pourciau (1993), qui observe également des accruals négatifs en T-1 sur un échantillon de départs non planifiés. Selon elle, les dirigeants auraient anticipé la gestion du résultat dès $\mathrm{T}-2$, les difficultés devenant impossible à masquer en T-1. D'ailleurs, on note parmi les départs forcés une différence significative au seuil de $5 \%$ entre les accruals discrétionnaires mesurés en T-2 et ceux mesurés en T. 
Tableau 5

Analyse longitudinale des accruals discrétionnaires

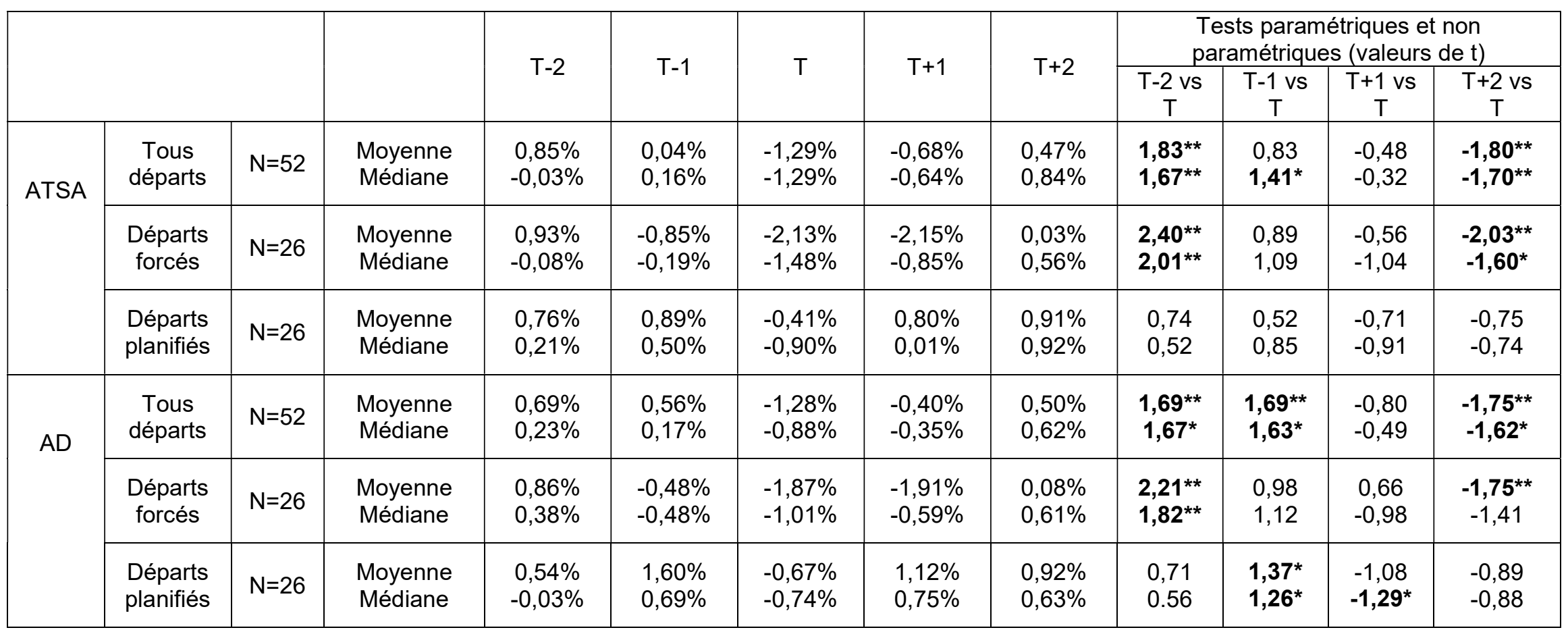

ATSA est le montant total des accruals totaux (hors dotations aux amortissements).

AD est le montant des accruals discrétionnaires calculé à partir du modèle global.

** Indique une significativité au seuil de $5 \%$ du test paramétrique t pour les moyennes et du test non paramétrique de comparaison des rangs de Wilcoxon pour les médianes (tests unilatéraux)

* Indique une significativité au seuil de $10 \%$ du test paramétrique t pour les moyennes et du test non paramétrique de comparaison des rangs de Wilcoxon pour les médianes (tests unilatéraux). 
Ces résultats indiquent qu'il existe une rupture en matière de choix comptables à l'occasion du changement de dirigeant. Durant l'exercice $\mathrm{T}+1$, on observe une remontée des accruals discrétionnaires. Néanmoins, en séparant les firmes selon la nature du départ comme le propose la figure 1, il apparaît que cette remontée ne concerne que les départs planifiés. En revanche, parmi les départs forcés, les accruals discrétionnaires restent négatifs en $\mathrm{T}+1$, semblant mettre en évidence des difficultés durables pour ces sociétés. Par conséquent, la rupture attendue entre $T$ et $T+1$ n'est vérifiée que parmi les firmes où le départ du dirigeant est planifié. Dans le cas des départs forcés, ce n'est qu'en $\mathrm{T}+2$ que les accruals discrétionnaires remontent de façon significative par rapport à l'année T (tests paramétriques significatifs au seuil de 5\%), tout en restant inférieurs aux accruals mesurés parmi les départs planifiés.

En conclusion, on observe bien une rupture dans l'évolution des accruals discrétionnaires. L'amplitude et la durée de la baisse des accruals dépendent de la nature du départ. Dans le cas du départ planifié, la chute des accruals est concentrée sur l'année T alors qu'elle s'étale sur trois exercices pour le départ forcé. D'autre part, l'intensité de la liquidation des pertes est de moindre importance dans le cas du départ planifié (-0.67\% contre $-1.87 \%$ pour le départ forcé).

Cependant, il est difficile de savoir si les résultats obtenus s'expliquent par une liquidation des pertes en $\mathrm{T}$, une gestion à la hausse du résultat en $\mathrm{T}+1$, ou les deux à la fois. Les deux phénomènes ne sont pas indépendants, dans la mesure où les accruals sont en partie réversibles. Ainsi, une gestion à la hausse des accruals antérieurement au changement de dirigeant influencera à la baisse les accruals de la période suivante. Cet effet est contrôlé de 
deux manières dans notre étude : la prise en compte des accruals décalés dans la régression et l'utilisation d'un échantillon de contrôle.

\subsection{Comparaison des accruals discrétionnaires de l'échantillon avec ceux de l'échantillon de contrôle}

La méthode consiste à comparer les accruals discrétionnaires de l'échantillon d'étude avec ceux de l'échantillon de contrôle sur la période [T-2, $\mathrm{T}+2]$ entourant le changement. Afin d'effectuer cette comparaison, les données de l'échantillon de contrôle ont été ajustées en fonction de la répartition temporelle et sectorielle observée au sein de l'échantillon de changements de dirigeants. Nous avons pondéré les accruals discrétionnaires de l'échantillon de contrôle en fonction des années des changements de dirigeants. Cette pondération des années de l'échantillon de contrôle est glissante en fonction des années de changement de dirigeant des sociétés étudiées ${ }^{10}$. Ensuite, les accruals discrétionnaires de chaque secteur d'activité ont été pondérés par la proportion de firmes du secteur au sein de l'échantillon de changement de dirigeants (8/52 par exemple pour le secteur 1). Enfin, compte tenu de la spécificité des firmes de l'indice CAC 40 et de leur surreprésentation au sein de l'échantillon de changement de dirigeants (14 sur 52), nous avons séparé, au sein de l'échantillon de contrôle, les sociétés du CAC 40 des autres firmes. Les accruals discrétionnaires mesurés au sein des deux sous échantillons (CAC 40 et non CAC 40) ont ensuite été pondérés par les proportions de firmes CAC $40(14 / 56)$ et non CAC $40(38 / 56)$ observées au sein de l'échantillon d'étude.

On obtient ainsi des accruals discrétionnaires moyens attendus, calculés à partir l'échantillon de contrôle, pour les années T-2 à T+2, que l'on peut comparer avec les accruals discrétionnaires des sociétés étudiées $\mathrm{AD}^{11}$ (tableau 6). Les accruals discrétionnaires sont en moyenne légèrement négatifs pour les sociétés de l'échantillon de contrôle et relativement stables sur la période d'étude [T-2; T+2]. En revanche, comme nous l'avons vu 
précédemment, les accruals discrétionnaires de l'échantillon des entreprises ayant connu un changement de dirigeant en $\mathrm{T}$ varient de façon importante sur la même période.

Tableau 6

Comparaison des accruals discrétionnaires de l'échantillon avec ceux de l'échantillon de contrôle

\begin{tabular}{|c|c|c|c|c|c|c|}
\hline Années $\epsilon$ & t nature du départ & $\begin{array}{c}\text { Échantillon de } \\
\text { changements } \\
\text { de dirigeant }\end{array}$ & $\begin{array}{l}\text { Échantillon de } \\
\text { changements } \\
\text { de dirigeant }\end{array}$ & $\begin{array}{c}\begin{array}{c}\text { Échantillon de } \\
\text { contrôle }\end{array} \\
508\end{array}$ & $\begin{array}{c}\text { t- test } \\
\text { significatif à }\end{array}$ & $\begin{array}{c}\text { Hypothès } \\
\text { es et }\end{array}$ \\
\hline & Tous départs & $\mathrm{N}=52$ & $0,69 \%$ & & $1,31^{*}$ & \\
\hline$A D(T-2)$ & Départs forcés & $\mathrm{N}=26$ & $0,86 \%$ & $-0,18 \%$ & 1,05 & $\mathrm{H}_{1}(+)$ \\
\hline & Départs planifiés & $\mathrm{N}=26$ & $0,54 \%$ & & 0,72 & \\
\hline & Tous départs & $\mathrm{N}=52$ & $0,56 \%$ & & $1,16^{*}$ & \\
\hline$A D(T-1)$ & Départs forcés & $\mathrm{N}=26$ & $-0,48 \%$ & $-0,20 \%$ & $-0,30$ & $\mathrm{H}_{1}(+)$ \\
\hline & Départs planifiés & $\mathrm{N}=26$ & $1,60 \%$ & & $1,94^{* *}$ & \\
\hline & Tous départs & $\mathrm{N}=52$ & $-1,28 \%$ & & $-1,34^{*}$ & \\
\hline$A D(T)$ & Départs forcés & $\mathrm{N}=26$ & $-1,87 \%$ & $-0,25 \%$ & $-1,76^{* *}$ & $\mathrm{H}_{2}(-)$ \\
\hline & Départs planifiés & $\mathrm{N}=26$ & $-0,67 \%$ & & $-0,45$ & \\
\hline & Tous départs & $\mathrm{N}=52$ & $-0,40 \%$ & & $-0,23$ & \\
\hline $\mathrm{AD}(\mathrm{T}+1)$ & Départs forcés & $\mathrm{N}=26$ & $-1,92 \%$ & $-0,24 \%$ & $-1,87$ & $\mathrm{H}_{3}(+)$ \\
\hline & Départs planifiés & $\mathrm{N}=26$ & $1,12 \%$ & & $1,50^{*}$ & \\
\hline & Tous départs & $\mathrm{N}=52$ & $0,50 \%$ & & $1,40^{*}$ & \\
\hline $\mathrm{AD}(\mathrm{T}+2)$ & Départs forcés & $\mathrm{N}=26$ & $0,08 \%$ & $-0,30 \%$ & 0,91 & $\mathrm{H}_{3}(+)$ \\
\hline & Départs planifiés & $\mathrm{N}=26$ & $0,92 \%$ & & $1,38^{*}$ & \\
\hline
\end{tabular}

AD est le montant des accruals discrétionnaires calculé à partir du modèle global.

** Indique une significativité au seuil de $5 \%$ du test paramétrique t pour les moyennes (test unilatéral).

* Indique une significativité au seuil de $10 \%$ du test paramétrique t pour les moyennes (test unilatéral).

Tout d'abord, le test reporté dans le tableau 6 indique que les accruals discrétionnaires mesurés en T-2 et T-1 au sein de l'échantillon de changement de dirigeant sont supérieurs à ceux de l'échantillon de contrôle. La différence entre les moyennes est significative au seuil de $10 \%$ en $\mathrm{T}-2$ et $\mathrm{T}-1$. Ces résultats accréditent l'hypothèse $\mathrm{H}_{1}$, selon laquelle les dirigeants tendent à gérer à la hausse les résultats comptables avant un changement. Ces résultats sont 
conformes à ceux obtenus par Dechow et Sloan (1991) et Reitenga et Tearney (2003). Par ailleurs, en séparant les firmes en fonction de la nature du départ, on constate que pour les départs forcés la gestion du résultat s'opère en T-2, alors que pour les départs planifiés la gestion maximale s'effectue sur l'exercice T-1 juste antérieur au départ.

Ensuite, durant l'exercice $\mathrm{T}$ du changement, on constate que les accruals discrétionnaires sont négatifs et inférieurs à ceux mesurés au sein de l'échantillon de contrôle, conformément à l'hypothèse $\mathrm{H}_{2}$. Les différences observées sont significatives au seuil de $10 \%$ pour l'échantillon global et plus particulièrement parmi le sous-échantillon des départs forcés, à l'instar des résultats obtenus par Pourciau (1993), Murphy et Zimmerman (1993) et Wells (2002). Enfin, durant les exercices $\mathrm{T}+1$ et $\mathrm{T}+2$, les résultats varient en fonction de la nature du départ. Parmi les départs planifiés, les accruals discrétionnaires sont significativement supérieurs à ceux de l'échantillon de contrôle pour les deux années qui suivent le changement, ce qui conforte l'hypothèse $\mathrm{H}_{3}$. En revanche, dans le cas des départs forcés, l'hypothèse n'est pas vérifiée. On constate même en $\mathrm{T}+1$ des accruals inférieurs à ceux l'échantillon de contrôle. Ces résultats vont à l'encontre de ceux obtenus par Godfrey et al. (2003), qui observent une gestion haussière plus forte en cas de départ forcé.

\subsection{L'influence de la nature du départ}

Afin d'évaluer l'impact de la nature du départ sur les politiques comptables dans le contexte d'un changement de dirigeant, le tableau 7 présente les tests de différences entre les accruals discrétionnaires mesurés au sein des deux sous-échantillons de départs forcés et planifiés. 
Tableau 7

Comparaison des accruals discrétionnaires selon la nature du départ (forcé/planifié)

\begin{tabular}{|c|c|c|c|c|c|c|}
\hline & & $\begin{array}{l}\text { Départs } \\
\text { forcés } \\
(\mathrm{N}=26)\end{array}$ & $\begin{array}{c}\text { Départs } \\
\text { planifiés } \\
(\mathrm{N}=26)\end{array}$ & $\begin{array}{c}\text { Statistique } \\
\mathbf{t} \\
\text { (valeur } \\
\text { de } \mathbf{t} \text { ) }\end{array}$ & $\begin{array}{c}\text { Statistique } \\
\text { de } \\
\text { Wilcoxon } \\
\text { (z-score) }\end{array}$ & $\begin{array}{c}\text { Hypothèses } \\
\text { et signes } \\
\text { attendus }\end{array}$ \\
\hline$A D(T-2)$ & $\begin{array}{l}\text { Moyenne } \\
\text { Médiane }\end{array}$ & $\begin{array}{l}0,85 \% \\
0,38 \% \\
\end{array}$ & $\begin{array}{r}0,54 \% \\
-0,03 \% \\
\end{array}$ & 0,246 & 0,433 & $\mathrm{H}_{4 a}(+)$ \\
\hline$A D(T-1)$ & $\begin{array}{l}\text { Moyenne } \\
\text { Médiane }\end{array}$ & $\begin{array}{l}-0,48 \% \\
-0,48 \% \\
\end{array}$ & $\begin{array}{l}1,60 \% \\
0,69 \% \\
\end{array}$ & $-1,66$ & $-0,96$ & $\mathrm{H}_{4 \mathrm{a}}(+)$ \\
\hline$A D(T)$ & $\begin{array}{l}\text { Moyenne } \\
\text { Médiane }\end{array}$ & $\begin{array}{l}-1,87 \% \\
-1,01 \% \\
\end{array}$ & $\begin{array}{l}-0,67 \% \\
-0,74 \%\end{array}$ & $-0,79$ & $-0,73$ & $\mathrm{H}_{4 b}(-)$ \\
\hline$A D(T+1)$ & $\begin{array}{l}\text { Moyenne } \\
\text { Médiane }\end{array}$ & $\begin{array}{l}-1,92 \% \\
-0,59 \%\end{array}$ & $\begin{array}{l}1,12 \% \\
0,75 \%\end{array}$ & $-2,35$ & $-2,24$ & $\mathrm{H}_{4 \mathrm{c}}(+)$ \\
\hline$A D(T+2)$ & $\begin{array}{l}\text { Moyenne } \\
\text { Médiane }\end{array}$ & $\begin{array}{l}0,08 \% \\
0,61 \% \\
\end{array}$ & $\begin{array}{l}0,92 \% \\
0,63 \% \\
\end{array}$ & $-0,77$ & $-1,05$ & $\mathrm{H}_{4 \mathrm{c}}(+)$ \\
\hline
\end{tabular}

AD est le montant des accruals discrétionnaires calculé à partir du modèle global.

** Indique une significativité au seuil de $5 \%$ du test paramétrique t pour les moyennes et du test non paramétrique de comparaison des rangs de Wilcoxon pour les médianes (tests unilatéraux).

* Indique une significativité au seuil de $10 \%$ du test paramétrique t pour les moyennes et du test non paramétrique de comparaison des rangs de Wilcoxon pour les médianes (tests unilatéraux).

Sur ce point, nous avons supposé que la gestion du résultat par les dirigeants sortants et entrants est plus forte en cas de départ forcé. Les résultats ne semblent pas confirmer les hypothèses émises. En T-2, la différence observée va dans le sens attendu par l'hypothèse $\mathrm{H}_{4 \mathrm{a}}$ mais n'est pas significative. En T-1, la relation est inverse à celle attendue, les dirigeants dont le départ est planifié semblant être davantage en mesure d'exercer leur discrétion sur les résultats. Durant l'année T de succession, les accruals discrétionnaires sont plus largement négatifs en cas de départ forcé qu'en cas de départ planifié, conformément à l'hypothèse $\mathrm{H}_{4 b}$ et aux travaux de Murphy et Zimmerman (1993) et Wells (2002). Cependant, la différence mesurée entre les deux sous-échantillons n'est pas significative. En $\mathrm{T}+1$, on observe une relation inverse à celle attendue $\left(\mathrm{H}_{4 \mathrm{c}}\right)$. En effet, la gestion du résultat à la hausse ne semble concerner que le sous-échantillon des départs planifiés, les autres firmes enregistrant, comme en $\mathrm{T}$, des accruals largement négatifs. Enfin, en $\mathrm{T}+2$ les accruals redeviennent positifs pour les départs forcés, mais ils sont moins élevés que pour les départs planifiés, contrairement à l'hypothèse émise. En définitive, il semblerait que la marge de manœuvre en matière de 
gestion à la hausse du résultat soit limitée pour les dirigeants des firmes du sous-échantillon des départs forcés.

\subsection{L'influence des facteurs de gouvernance}

Afin de compléter l'analyse, les hypothèses 4 et 5 sont testées à partir d'une régression dans laquelle nous introduisons comme variables indépendantes la nature du départ, ainsi que des variables de gouvernance et de contrôle. Le modèle de régression linéaire, dont la variable expliquée est le montant des accruals discrétionnaires, est le suivant :

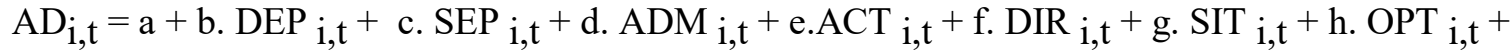

$$
\begin{aligned}
& +\mathrm{j} \cdot \text { TAIL }_{\mathrm{i}, \mathrm{t}}+\mathrm{k} \cdot \mathrm{END}_{\mathrm{i}, \mathrm{t}}+\mathrm{l}_{\mathrm{ADL}} \mathrm{i,t}+\mathrm{m} \cdot \mathrm{FTE}_{\mathrm{i}, \mathrm{t}}+\varepsilon_{\mathrm{i}, \mathrm{t}}
\end{aligned}
$$

$\mathrm{AD}_{\mathrm{i}, \mathrm{t}} \quad$ est le montant des accruals discrétionnaires de la firme i durant l'exercice $\mathrm{t}(\mathrm{t}=\mathrm{T}-2 \mathrm{à} \mathrm{T}+2)$;

$\mathrm{DEP}_{i, t}$ est égal à 1 en cas de départ forcé, et à 0 sinon;

$\mathrm{SEP}_{\mathrm{i}, \mathrm{t}}$ est égal à 0 si les organes de contrôle et de gestion sont séparés, 1 sinon;

$\mathrm{ADM}_{\mathrm{i}, \mathrm{t}}$ est le pourcentage d'administrateurs indépendants dans le conseil de direction;

$\mathrm{ACT}_{\mathrm{i}, \mathrm{t}}$ est le pourcentage de droits de vote détenu par les trois principaux actionnaires;

DIR $_{i, t}$ est le pourcentage de droits de vote détenu par le dirigeant;

SIT i,t est égal à 1 si le dirigeant de la firme i conserve un poste d'administrateur après le changement, 0 sinon;

$\mathrm{OPT}_{\mathrm{i}, \mathrm{t}}$ est égal à 1 si le dirigeant de la firme i bénéficie d'options de souscription d'actions, 0 sinon;

TAIL $_{i, t}$ est le logarithme népérien du total de l'actif en début d'exercice $t$;

END $_{i, t}$ est le rapport dettes financières long terme en fin d'exercice t divisé par l'actif total en début d'exercice;

ADL i,t est le montant des accruals discrétionnaires de la firme i de l'exercice t-1 divisé par l'actif total de l'exercice t-2;

FTE $_{i, t}$ est le montant des flux de trésorerie d'exploitation de la firme i de l'exercice $t$ divisé par l'actif total de début d'exercice. 
Les régressions ont été menées pour chaque exercice de la période $\mathrm{T}-2$ à $\mathrm{T}+2$. Les observations dont les résidus excèdent trois écart-types ont été exclues de l'analyse. Les facteurs d'inflation de la variance (VIF) inférieurs à 2,5 indiquent l'absence de problème de multi-colinéarité. Les résultats sont présentés dans le tableau 8.

Tableau 8

Tests de régression de facteurs de gestion de résultat

\begin{tabular}{|cccccc|}
\hline & $\begin{array}{c}\text { Régression } \\
\mathbf{T}-\mathbf{2}\end{array}$ & $\begin{array}{c}\text { Régression } \\
\mathbf{T - 1}\end{array}$ & $\begin{array}{c}\text { Régression } \\
\mathbf{T}\end{array}$ & $\begin{array}{c}\text { Régression } \\
\mathbf{T + 1}\end{array}$ & $\begin{array}{c}\text { Régression } \\
\mathbf{T + 2}\end{array}$ \\
\hline $\mathrm{N}=$ & 49 & 47 & 50 & 50 & 50 \\
\hline $\mathrm{R}^{2}=$ & 0,297 & 0,431 & 0,240 & 0,219 & 0,078 \\
\hline $\mathrm{F}=$ & $2,841^{* * *}$ & $4,170^{* * *}$ & $2,409^{* *}$ & $2,246^{* *}$ & 1,375 \\
\hline constante & $-0,153^{* *}$ & $-0,101^{*}$ & $-0,059$ & $-0,008$ & 0,087 \\
DEP & 0,004 & $-0,024^{* * *}$ & $-0,019$ & $-0,037^{*}$ & $-0,007$ \\
\hline SEP & $-0,012$ & $-0,002$ & $-0,007$ & 0,002 & 0,019 \\
ADM & $-0,030$ & $0,060^{* * *}$ & $0,106^{* * *}$ & 0,028 & $0,059^{*}$ \\
\hline ACT & 0,007 & $0,029^{*}$ & 0,014 & $-0,007$ & 0,018 \\
DIR & 0,003 & 0,047 & $0,124^{* * *}$ & $-0,020$ & 0,056 \\
\hline SIT & $-0,003$ & $-0,017^{*}$ & $-0,021$ & $-0,017$ & $-0,002$ \\
OPT & $-0,002$ & $-0,002$ & 0,001 & 0,009 & $-0,010$ \\
\hline TAIL & $0,010^{* * *}$ & 0,004 & 0,000 & 0,001 & $-0,006$ \\
END & $-0,104^{* * *}$ & $0,066^{* * *}$ & 0,015 & 0,032 & 0,041 \\
ADL & 0,124 & $-0,103$ & 0,143 & $0,318^{* *}$ & $0,253^{* *}$ \\
FTE & $-0,248^{* * *}$ & $-0,257^{* * *}$ & 0,078 & $-0,166$ & $-0,023$ \\
\hline
\end{tabular}

Les signes *,**,*** indiquent une significativité aux seuils respectifs de $10 \%, 5 \%$ et $1 \%$.

DEP est égal à 1 en cas de départ forcé, et à 0 sinon, SEP est égal à 0 si les organes de contrôle et de gestion sont séparés, 1 sinon, ADM est le pourcentage d'administrateurs indépendants dans le conseil de direction, ACT est le pourcentage de droits de vote détenu par les trois principaux actionnaires, DIR est le pourcentage de droits de vote détenu par le dirigeant, SIT est égal à 1 si le dirigeant conserve un poste d'administrateur après le changement, 0 sinon, OPT est égal à $1 \mathrm{si}$ le dirigeant bénéficie d'options de souscription d'actions, 0 sinon, TAlL est le logarithme népérien du total de l'actif en début d'exercice, END est le rapport dettes financières long terme en fin d'exercice divisé par l'actif total en début d'exercice, ADL correspond aux accruals discrétionnaires de l'année précédente, FTE est le montant des flux de trésorerie d'exploitation de l'exercice divisé par l'actif total de début d'exercice.

Les R-deux ajustés varient de 7,8\% pour $\mathrm{T}+2$ à $43,1 \%$ en $\mathrm{T}-1$, année avant le départ et dont les accruals semblent le mieux expliqués par le modèle. Le test de Fisher indique que les régressions sont significatives à un seuil de $1 \%$ pour $\mathrm{T}-2$ et $\mathrm{T}-1,5 \%$ pour $\mathrm{T}$ et $\mathrm{T}+1$. En revanche, la régression en $\mathrm{T}+2$ apparaît peu significative. 
Tout d'abord, la nature du départ influence les accruals autour du changement. Un départ forcé influence à la baisse ces derniers entre $\mathrm{T}-1$ et $\mathrm{T}+2$ (signe négatif dans la régression). La variable est significative en $\mathrm{T}-1$ (au seuil de $1 \%$ ) et en $\mathrm{T}+1$ (au seuil de $10 \%$ ), ce qui est cohérent avec les résultats des tests univariés.

Les facteurs de gouvernance influencent plus particulièrement les accruals des années T-1 et $\mathrm{T}$ qui sont les années charnières du changement. Durant l'exercice $\mathrm{T}$, la présence d'administrateurs indépendants $(\mathrm{ADM})$ et la détention d'actions par le dirigeant (DIR) semblent limiter l'intensité de la baisse des accruals. Conformément au rapport Bouton, la présence d'administrateurs indépendants dans le conseil permettrait de mieux contrôler les dirigeants dans leurs décisions comptables et de gestion ${ }^{12}$. De même, lorsque les dirigeants entrants sont actionnaires, un mécanisme d'auto-contrôle tend à limiter les comportements opportunistes.

En T-1, on constate un lien positif entre les accruals et à la fois la présence d'administrateurs indépendants et la concentration de l'actionnariat. Dans la mesure où, dès T-1, un certain nombre de firmes voient leurs accruals chuter pour des raisons de performance, on peut penser que les firmes à forte structure de gouvernance auront moins tendance à enregistrer des accruals négatifs. Par ailleurs, les dirigeants qui conservent un poste d'administrateur ont une propension à limiter les accruals discrétionnaires en T-1. Ce résultat, contraire à celui obtenu par Reitenga et Tearney (2003), peut s'expliquer par le contexte de cette étude. En effet, plusieurs changements correspondent à une transition du dirigeant sortant vers un poste de président de l'organe de contrôle, dont il a lui-même décidé la forme. Dans ce cas, qui correspond en général à un départ planifié, la performance à court-terme n'est pas un critère déterminant dans la nomination comme président de l'organe de contrôle. 
Les variables de contrôle mettent en évidence en T-2 et T-1 une incitation nette à la gestion à la hausse lorsque la performance est faible. Les variables taille (en T-1) et endettement (en T1 et T-2) sont également significatives mais difficiles à interpréter. D'autre part, le lien positif entre les accruals d'une année sur l'autre en $\mathrm{T}+1$ et $\mathrm{T}+2$ semble indiquer la présence de composantes à long terme (e.g. reprises sur provisions) parmi les variables comptables observées après le changement du dirigeant.

\section{Conclusion}

Notre recherche vise à étudier empiriquement les pratiques comptables au sein des entreprises durant les exercices qui entourent l'exercice T d'un changement de dirigeant. A partir d'un échantillon de 52 changements de dirigeant au sein d'entreprises françaises cotées entre 2000 et 2005, nous étudions l'évolution des choix comptables effectués par les dirigeants sortants et entrants sur une période de cinq ans autour de l'exercice de succession. Tout d'abord, une analyse longitudinale indique une baisse significative des accruals durant l'exercice $\mathrm{T}$ du changement, suivie d'une remontée après l'exercice de succession. Cependant, dans le cas des départs forcés, la baisse des accruals se produit dès l'exercice T-1, et la remontée attendue ne se produit qu'en $\mathrm{T}+2$. Cette première analyse montre que l'intensité et la durée de la baisse des accruals dépendent de la nature du départ.

Ensuite, nous avons comparé les accruals discrétionnaires observés à ceux d'un échantillon de contrôle représentatif, sur le plan temporel et sectoriel, de notre échantillon de changements de dirigeants. Cette méthode nous semble appropriée pour tenir compte de l'influence de la conjoncture sur les accruals. En comparant les accruals discrétionnaires mesurés au sein des deux échantillons, il apparaît que les dirigeants sortants ont tendance à 
gérer à la hausse les résultats durant les deux exercices qui précèdent leur départ. Cette gestion se concentre sur l'exercice T-2 pour les départs forcés, alors que dans le cas des départs planifiés la gestion maximale s'observe durant l'exercice T-1 juste antérieur au départ. Une baisse significative des accruals sur l'exercice $\mathrm{T}$ semble aussi vérifiée, plus particulièrement dans le cas des départs forcés. La gestion à la hausse du résultat après l'exercice de succession, en $\mathrm{T}+1$ et $\mathrm{T}+2$, n'est observée que parmi les départs planifiés de dirigeants. L'analyse multivariée confirme l'incidence de la nature du départ sur les accruals et montre l'influence des administrateurs indépendants et de la structure de l'actionnariat sur l'ampleur de la baisse des accruals. Cependant, les dirigeants restant comme administrateurs après le changement semblent moins incités à la gestion du résultat la dernière année de leur mandat. Toutefois, le caractère opportuniste de l'action des dirigeants sur les accruals reste délicat à évaluer.

Cette étude met ainsi en évidence l'influence du contexte du départ sur les variables comptables autour d'un changement de dirigeant. La baisse des accruals se concentre sur un exercice en cas de départ planifié et semble traduire l'effet ponctuel des décisions prises par le nouveau dirigeant. En revanche, en cas de départ forcé, les accruals apparaissent durablement négatifs puisque la période dépressive s'étale sur trois exercices. Cette phase semble refléter les difficultés rencontrées par les firmes. Dans ce contexte, les caractéristiques de gouvernance, comme la présence d'administrateurs indépendants et/ou de dirigeants actionnaires, sont susceptibles de prévenir de telles difficultés.

Dans le prolongement de cette recherche, il serait intéressant d'analyser, au-delà de la distinction entre départ forcé et planifié et des variables de gouvernance, d'autres facteurs qui influencent les politiques comptables des dirigeants. Ainsi, la prise en compte des caractéristiques du dirigeant telles que la durée du mandat ou sa formation pourraient faire 
l'objet d'études ultérieures. Par ailleurs, une analyse plus approfondie des composants des accruals permettrait de mieux de mieux comprendre la nature et les motivations des choix comptables opérés par les dirigeants. 


\section{Annexes}

Annexe 1. Nom des sociétés ayant connu un changement de dirigeant pendant la période

\begin{tabular}{|ll|}
\hline A NOVO SA & Groupe Partouche \\
Accor SA & Havas \\
Air Liquide & Hubwoo \\
Alstom & IMS \\
Altran Tech & Info Vista \\
Bacou Dalloz & Ingenico \\
Buffalo Grill & Lafarge \\
Bull & Lagardere \\
Canal Plus SA & Medidep \\
Cap Gemini SA & NRJ Group \\
Carrefour & Onet \\
Casino & Otor \\
Club Med & PPR \\
Cofiroute & Renault SA \\
EDF & Rexel \\
Eramet & Rhodia SA \\
Esso S A F & Samse NV \\
Euro Disney SCA & Sanef \\
Eurotunnel & SR Téléperformance \\
Evialis & STMicroelectron \\
France TIcm & THOMSON \\
Gascogne & Unilog \\
Gaumont & Vallourec \\
Gaz de France & Vivendi \\
Gemplus & Wavecom \\
Go Sport & \\
\hline
\end{tabular}

Annexe 2.

Répartition sectorielle des sociétés ayant connu un changement de dirigeant pendant la période

1- Matières premières, énergie et eau

Nombre firmes

2- Industrie

8

3- Biens de consommation

4- Santé

1

5- Services-Communication

2

6- Technologie

Total

52




\section{Bibliographie}

Aboody, D., Kasznik, R. (2000). CEO Stock Option Awards and the Timing of Corporate Voluntary Disclosures. Journal of Accounting and Economics 29(1): 73-100.

Alexandre, H., Paquerot, M. (2000). Efficacité des structures de contrôle et enracinement des dirigeants. Finance-Contrôle-Stratégie 3(2) : 5-29.

Beneish, M. (1998). Discussion of "Are accruals during initial public offerings opportunistic?" Review of Accounting Studies 3 (1 et 2): 209-221.

Bergstresser, D., Philippon, T., (2006). CEO Incentives and Earnings Management. Journal of Financial Economics 80(3): 511-529.

Bozec Y., (2008). Concentration de l'actionnariat, séparation des droits de vote et des droits de propriété et gestion des bénéfices: une étude canadienne. Revue canadienne des Sciences de l'Administration 25 : 67-82.

Burns, N., Kedia, S. (2006). The Impact of Performance-Based Compensation on Misreporting. Journal of Financial Economics 79(1): 35-67.

Caby, J., Hirigoyen, G. (2005). Création de valeur et gouvernance de l'entreprise. Economica.

Conyon, M., Florou, A. (2006). The pattern of investment surrounding CEO retirements: UK evidence. The British Accounting Review 38: 299-319.

Coughlan, A.T., Smith, R.M. (1985). Executive compensation, management turnover, and firm performance: an empirical investigation. Journal of Accounting and Economics 7: 43-66.

DeAngelo, L. (1988). Managerial competition, information costs and corporate governance: the use of accounting performance measures of proxy contests. Journal of Accounting and Economics 10: 3-36.

Dechow, P. (1994). Accounting earnings and cash-flows as measures of firm performance: The role of accounting accruals. Journal of Accounting and Economics 18: 3-42.

Dechow P., Sloan, R. (1991). Executives incentives and the horizon problem: An empirical investigation. Journal of Accounting and Economics 14: 51-89.

Dechow P., Sloan, R., Sweeney, A. (1995). Detecting Earnings Management. The Accounting Review. 70(2): 193-225.

DeFond, M., Jiambalvo, J. (1994). Debt-covenant violations and manipulation of accruals. Journal of Accounting and Economics 17: 145-176.

Desai, H., Hogan, C., Wilkins, M., (2006). The Reputational Penalty for Aggressive Accounting: Earnings Restatements and Management Turnover. The Accounting Review 81(1): 83-112.

Dherment-Férère, I. (1999). Changements de dirigeant et richesse des actionnaires : une analyse explicative multivariée. Finance-Contrôle-Stratégie 2: 71-97.

Engel, E., Hayes, R., Wang, X. (2003). CEO turnover and properties of accounting information. Journal of Accounting and Economics 36: 197-226. 
Fama, E. (1980). Agency problems and the theory of the firm. Journal of Political Economy 2: 288-307.

Fama, E., Jensen, M. (1983). Separation of Ownership and Control. Journal of Law and Economics 26: 301-326.

Farrell, K., Whidbee, D. (2003). Impact of firm performance expectations on CEO turnover and replacement decisions. Journal of Accounting and Economics 36: 165-196.

Francis, J., Hanna, D., Vincent, L. (1996). Causes and effects of discretionary asset write-offs. Journal of Accounting Research 34: 117-134.

Gamson, W., Scotch, N. (1964). Scapegoating in baseball. The American Journal of Sociology 70: 69-76.

Godfrey, J., Mather, P., Ramsay, A. (2003). Earnings and impression management in financial reports: the case of CEO changes. Abacus 39 (1): 95-123.

Guan, L., Wright, C.J., Leikam, S.L. (2005). Earnings management and forced CEO dismissal. Advances in Accounting 21: 61-81.

Guest, R. (1962). Managerial succession in complex organizations. The American Journal of Sociology 68: 47-54

Hermalin, B., Weisbach, M. (1998). Endogenously chosen boards of directors and their monitoring of the CEO. American Economic Review 88: 96-118.

Hunt, A., Moyer, S., Shevlin T. (1996). Managing interacting accounting measures to meet multiple objectives: A study of LIFO firms. Journal of Accounting and Economics 21 (3): 339-374.

Jones, J. (1991). Earnings management during import relief investigations. Journal of Accounting Research 29: 193-228.

Labelle, R., Schatt, A. (2005). Structure de propriété et communication financière des entreprises françaises. Finance-Contrôle-Stratégie $8: 77-104$

Lasalle, R. (1990). The effect of CEO tenure on earnings management: theory and preliminary evidence. WP, Rutgers University.

Lasalle, R., Jones, S., Jain, R. (1993). The association between executive succession and discretionary accounting changes: earnings management or different perspectives. Journal of Business Finance and Accounting 20 (5): 653-671.

Karlsson, P., Neilson, G., Webster, J.-C. (2008). CEO succession 2008, the performance paradox. Strategy+Business 51 .

Maijoor, S., Vanstraelen, A. (2006). Earnings Management within Europe: the Effects of Member State Audit Environment, Audit Firm Quality and International Capital Markets. Accounting and Business Research 36(1): 169-193.

Mard, Y., Marsat, S. (2008). Les stratégies comptables précédant un changement de dirigeant en France. Finance Contrôle Stratégie 11(4): 111-136.

Moore, M. (1973). Management changes and discretionary accounting decisions. Journal of Accounting Research 11: 100-107.

Murphy, K., Zimmerman, J. (1993). Financial performance surrounding CEO turnover. Journal of Accounting and Economics 16: 273-316. 
Peasnell K., Pope P., Young S. (2005). Board monitoring and earnings management: do outside directors influence abnormal accruals? Journal of Business Finance and Accounting 32(7-8): 1311-1346

Pichard-Stamford, J.P. (1997). La contribution des réseaux des administrateurs à la légitimation $d u$ dirigeant auprès de multiples catégories d'actionnaires. Thèse de doctorat en sciences de Gestion à l'Université Bordeaux IV.

Pourciau, S. (1993). Earnings management and nonroutine executive changes. Journal of Accounting and Economics 16: 317-336.

Reitenga, A., Tearney, M. (2003). Mandatory CEO retirements, discretionary accruals, and corporate governance mechanisms. Journal of Accounting, Auditing and Finance 18:255-279.

Shleifer, A, Vishny, R. (1989). Management entrenchment: the case of manager specific investments. Journal of Financial Economics 25: 123-139.

Teoh, S., Welch, I., et Wong, T. (1998). Earnings management and the long run market performance of initial public offerings. The Journal of Finance 53 (6): 1935-1974.

Warner, J., Watts, R., Wruck, K. (1988). Stock prices and top management changes. Journal of Financial Economics 20: 461-492.

Watts, R., Zimmerman, J. (1986). Positive Accounting Theory. Englewood Cliffs, NJ: Prentice-Hall.

Weisbach, M.S. (1988). Outside directors and CEO turnover. Journal of Financial Economics 20: $432-460$.

Wells, P. (2002). Earnings management surrounding CEO changes. Accounting and Finance 42: 169-193. 


\section{Notes}

${ }^{1}$ Cette hypothèse de liquidation des pertes sur l'exercice de succession est connue en anglais sous le nom d'hypothèse du « big bath ».

${ }^{2}$ Les travaux de Dherment-Férère (1999) portent sur la réaction du marché français à l'annonce d'un changement de dirigeant. L'étude de Mard et Marsat (2008) s'intéresse aux stratégies comptables antérieures au changement de dirigeant.

${ }^{3}$ Les études empiriques sur le sujet donnent des résultats mitigés et peuvent conduire, selon les circonstances, à valider l'une des trois hypothèses.

${ }^{4}$ En outre, Godfrey et al. (2003) observent que la gestion du résultat s'accompagne d'une manipulation des graphiques de présentation des principales variables financières.

${ }^{5}$ Les sociétés financières (banques, sociétés d'assurance) et immobilières ont été exclues de l'échantillon en raison de leurs spécificités comptables.

${ }^{6} \mathrm{La}$ liste des sociétés retenues figure en annexe 1. Cette liste comporte 51 sociétés, Vivendi ayant changé deux fois de dirigeant pendant la période d'étude.

${ }^{7}$ La répartition sectorielle des firmes de l'échantillon est détaillée en annexe 2.

${ }^{8}$ Dont la valeur absolue des accruals normalisés est supérieure à $30 \%$ ou la valeur absolue de la variation du chiffre d'affaires est supérieure à $100 \%$.

${ }^{9}$ Dans le tableau 3, des statistiques d'années différentes ont été agrégées volontairement, afin de faire apparaître l'évolution de ces variables autour du changement de dirigeant initié durant l'année $\mathrm{T}$ (précisée dans le tableau 1). Les années $\mathrm{T}-2$ à $\mathrm{T}+2$ ne correspondent pas à des années calendrier, mais cette présentation nous a semblé plus pertinente dans le cadre de l'étude.

${ }^{10}$ L'année T n'existe pas réellement au sein de l'échantillon de contrôle. Elle a été déterminée en fonction de la date de changement de dirigeant de l'échantillon d'étude afin d'éviter des biais liés à la conjoncture dans la comparaison. Les accruals de l'échantillon de contrôle vont prendre en compte plusieurs années afin d'intégrer la disparité temporelle des années de changement de dirigeants. Par exemple pour l'année T, les accruals de l'échantillon de contrôle en 2002, 2003, 2004, sont pondérés par le nombre de changements de dirigeants de ces années, c'est-à-dire 9, 11 et 21. Le choix des années 2002 à 2004 pour l'échantillon de contrôle correspond à une période centrale de notre étude et permet de couvrir pratiquement $60 \%$ des données sur l'ensemble de la période. Bien que cet échantillon ne soit pas exhaustif, on note une stabilité des accruals mesurés au sein de l'échantillon de contrôle (de $-0.18 \%$ à$0.30 \%$ ) qui ne serait pas significativement affectée par l'introduction d'autres années.

${ }^{11}$ Les deux mesures des accruals discrétionnaires $\mathrm{AD}$ et ADsect conduisant à des résultats très voisins, seuls les résultats obtenus avec la variable $\mathrm{AD}$ sont retranscris.

${ }^{12}$ Le changement de dirigeant semble d'ailleurs s'accompagner d'un renforcement de la structure de gouvernance. Par exemple, dans le cas des départs forcés, le pourcentage d'administrateurs indépendants augmente de $40 \%$ à $48 \%$ entre $\mathrm{T}-2$ et $\mathrm{T}+2$. 